\title{
The role of palliative medicine in the acute medical admitting unit
}

\author{
Authors: Katherine Buxton, Laura Eades, Shivani Soni and Catherine Urch
}

\section{Aims}

To assess the impact of proactive attendance at the acute admitting unit daily board round.

\section{Methods}

A 3-month prospective pilot was undertaken, with the palliative care team aiming to attend three out of the five acute midday board rounds held per week to prompt identification of appropriate patients who would benefit from palliative care review.

\section{Results}

The data collection period spanned 3 months from 15 September to 15 December 2014. During this time, a representative from the palliative care team attended the board round on 26 out of 65 occasions, a mean of twice per week (range zero to four). The referral numbers varied from day to day, with a mean of 1.3 new referrals per board round attended (range zero to four). $55 \%$ of patients referred had a malignant diagnosis. Dementia at $21 \%$ was the single largest non-malignant group referred. Reasons for referral included terminal care, advance care planning, direction of care discussions and symptom control. We did not routinely receive referrals from the post-take teams on the days that we did not attend the board round, nor were referrals held over until the following day. During the pilot phase, analysis of our usual case load numbers showed an increase of 1.4 referrals per week over and above the average number for the preceding two 3-month periods.

\section{Conclusions}

The data suggest that attendance at the acute board round is a needed and effective way of highlighting specialist palliative care need, as demonstrated by a lack of referrals from the acute teams on the non-attendance days. If we could attend all board rounds, this has the potential to generate six or seven new referrals per week. The qualitative data suggest that the acute teams proactively selected patients for referral with symptom control or terminal care needs, both of which are classical referral criteria. However, the presence of specialist palliative care, especially when the board round was consultant led, enabled a discussion about wider care planning issues such as advance care planning and treatment escalation plans, which are traditionally more challenging for the admitting teams. Education around this wider remit of specialist palliative care and the active challenging of difficult issues is a key component to board-round attendance. The implications generated from this pilot study include earlier involvement of palliative care within the admission journey and hence the ability to potentially influence treatment plans and length of stay. 